\title{
My job impacts my sleep: signs and symptoms of insomnia among healthcare workers
}

\author{
Soomi LEE ${ }^{1 *}$, Brian D. GONZALEZ ${ }^{2}$ and Brent J. SMALL ${ }^{1}$ \\ ${ }^{1}$ School of Aging Studies, University of South Florida, USA \\ ${ }^{2}$ Department of Health Outcomes and Behavior, Moffitt Cancer Center, USA
}

Received September 13, 2020 and accepted November 30, 2020

Published online in J-STAGE December 5, 2020

\begin{abstract}
Potential insomnia in healthcare workers is a public health concern as it may degrade the quality of patient care. We examined the prevalence of insomnia symptoms in healthcare workers and their perceived need for a sleep intervention. Participants were 62 nurses working full-time at a U.S. hospital. These nurses were asked about background characteristics, perceived stress, sleep concerns, and need for a sleep intervention. They also participated in 14-d ecological momentary assessment (EMA) and actigraphy sleep study. A qualitative analysis showed that the majority (92\%) of participants reported at least one sleep concern with insomnia-related concerns being most prevalent $(68 \%)$. Quantitative analyses indicated that those with insomnia-related concerns had higher perceived stress overall and lower EMA sleep sufficiency and sleep quality. Moreover, participants with insomnia concerns had shorter actigraphy-measured nap duration prior to non-workdays than those without. Nearly all $(\mathbf{9 5} \%)$ expressed interest in participating in a sleep intervention; an online format and mindfulness contents were most preferred. Our results suggest a high prevalence of insomnia symptoms and a high interest in a sleep intervention in nurses. Information obtained from this study could be used to deliver a tailored sleep intervention for nurses whose role in public health is essential.
\end{abstract}

Key words: Insomnia, Healthcare, Nurses, Perceived need for an intervention, Online sleep intervention, Mindfulness

\section{Introduction}

Good sleep allows us to recover physical and mental resources for the next day. Good sleep is needed for workers in any occupation, but may be especially important for healthcare workers who deliver direct care to patients. In particular, the role of nurses is critical as they have more opportunities to interact with patients and may influence health behaviors and rehabilitation of patients ${ }^{1-4)}$. However, the work conditions of nurses, such as extended work

*To whom correspondence should be addressed.

E-mail: soomilee@usf.edu

(C)2021 National Institute of Occupational Safety and Health shifts (e.g., 7 am-7 pm or 7 pm-7 am) and stress associated with proximity to life-threatening health conditions, may make them vulnerable to sleep problems. Insomnia is one of the most prevalent sleep problems, and insomnia in nurses is associated with burnout ${ }^{5,6}$, poor work productivity and absenteeism ${ }^{7)}$, and contribute to making errors and attentional failures while providing care to patients ${ }^{8)}$. Thus far, however, there is a lack of studies examining signs and symptoms of insomnia among U.S. nurses using detailed sources of sleep hygiene. The goal of this study is to examine the prevalence of insomnia symptoms in a sample of U.S. nurses. We also describe the perceived need and acceptability of a sleep intervention assessed by these nurses. 
The definition of insomnia varies by studies, resulting in different statistics about prevalence of insomnia. In the literature, four definitions of insomnia have been used: (1) insomnia symptoms (presence only or with frequency or severity quantifiers), (2) insomnia symptoms with daytime consequences, (3) dissatisfaction with sleep quality or quantity, and (4) insomnia diagnoses ${ }^{9)}$. The prevalence of insomnia ranges from $6 \%$ (insomnia diagnoses) to $48 \%$ (presence of insomnia symptoms) in general populations ${ }^{9,10)}$. Although specific criteria to define insomnia differ, most studies use common symptoms of insomnia suggested by the Diagnostic and Statistical Manual of Mental Disorders $(\mathrm{DSM}-5)^{11)}$. Those are difficulty initiating or maintaining sleep (including early morning awakenings) and nonrestorative sleep.

In U.S. working populations, $20-41 \%$ report having at least one of the insomnia symptoms based on the very basic definition and this prevalence is decreased to $11 \%$ when defined more restrictively with additional criteria of frequency (e.g., symptoms present at least a few nights week) and daytime impairment ${ }^{7}$. In a study on Norwegian nurses working extended shifts, the prevalence of insomnia symptoms (based on Bergen Insomnia Scale, which also requires both nighttime problems and daytime impairment) varies between $45-57 \%{ }^{12}$. To capture overall signs and symptoms of insomnia in U.S. nurses, this study uses the very basic definition that assesses the presence of insomnia symptoms without restrictive criteria.

Potential insomnia symptoms can be further verified by the individual's retrospective self-report, sleep diaries, and other objective methods such as actigraphy. Example insomnia-specific sleep characteristics include feeling unrested upon waking and being dissatisfied with their sleep quality despite ample time in bed ${ }^{11,13,14)}$. Individuals with insomnia may also take longer to fall asleep (i.e., longer sleep onset latency) and have frequent awakenings or more wake time after falling asleep ${ }^{15,16)}$. Nurses with insomnia symptoms may report higher perceived stress overall, because stress is considered the primary cause of insomnia ${ }^{17)}$. Worry about next day and anticipation of stress are also common among subjects with insomnia ${ }^{18,19)}$. Thus, nurses with insomnia symptoms may particularly exhibit insomnia-specific sleep characteristics on nights before workdays in the anticipation of stressful experiences at work. However, less is known about whether and how sleep characteristics differ between workdays and non-workdays in workers with potential insomnia versus those without potential insomnia. This approach may help understand the micro-level effect of work on insomnia symptoms and guide early detection of insomnia in workers who are often too busy to visit a clinic for diagnosis, like nurses.

In addition to measuring signs and symptoms of Insomnia, we assess nurses' perceived need for a sleep intervention and preferred delivery formats and contents. This is important to find ways to effectively improve insomnia symptoms in nurses, because sleep-focused interventions are sorely lacking in the field of occupational health in general $^{20)}$. Improving insomnia symptoms and overall sleep health may reduce interpersonal conflicts and psychosocial stressors and enhance daily vigilance and mood necessary for providing high-quality care ${ }^{21-24)}$. The nursing literature has focused mostly on improving patient outcomes but promoting nurses' sleep may have positive effects on patients as well. There are several efficacious treatments for insomnia, such as cognitive behavioral therapy for insomnia (CBT-I), but there is no existing insomnia intervention specifically designed for nurses. There is evidence that an intervention tailored for a target group is more effective ${ }^{25)}$. To design the most appealing and approachable form of a sleep intervention, this study collets data on nurses' preferences on possible delivery formats (e.g., online, group, or in-person) and contents of the intervention (e.g., CBT-I, sleep hygiene education, and mindfulness strategies).

The present study had three aims. The first aim examined sleep concerns reported by nurses, who are the largest group of the healthcare workforce and in close proximity to the delivery of care. We hypothesized that insomniarelated concerns would be prevalent in nurses. Additionally, we expected that participants with insomnia-related concerns would report higher perceived stress compared to those without insomnia concerns. The second aim compared sleep characteristics by the presence of insomniarelated concerns and by type of day (i.e., workday vs. non-workday). We hypothesized that, compared to those without insomnia concerns, participants with insomniarelated concerns would exhibit poorer sleep characteristics and such differences would be more apparent on nights before workdays. The third aim examined willingness to participate in a future sleep intervention and the preferred delivery formats and contents of the intervention in this sample. We hypothesized that interest in a sleep intervention would be higher in those with insomnia-related concerns than those without. 


\section{Methods}

\section{Participants and procedure}

Participants were inpatient nurses working at a cancer hospital in Florida. Inclusion criteria required participants to: (a) be at least $18 \mathrm{yr}$ of age, (b) work full-time, (c) provide direct, inpatient care to cancer patients or cancer survivors, (d) work with fixed 12-h day shifts (e.g., 7 am-7 pm) or fixed 12-h night shifts (e.g., 7 pm-7 am), and (e) possess a smartphone to use for app-based ecological momentary assessment (EMA) questions. Exclusion criteria included (a) working variable-, rotating-, or split- shifts or (b) not possessing a smartphone.

Participants who met the study criteria were invited to participate in the initial study meeting that was part of a regular nurse group meeting. During the initial meeting, the investigators explained the background of the study, study protocol, and participant compensation. Any interested nurses were instructed to place their names on a sign-up sheet. A series of study invitation emails was also distributed to the nurse group listserv. Trained research assistants scheduled individual informed consent meetings with those who signed up. Out of over 500 inpatient nurses working at the study site (including those who were ineligible), 62 who agreed to participate in the study (103\% of the recruitment target of 60). Informed consent meetings took place in the workplace during times preferred by participants. Following informed consent, all 62 participants completed a background survey assessing sociodemographic characteristics, work characteristics, perceived stress, and questions regarding sleep concerns and a future sleep intervention (see measures section below for details). The informed consent procedure and background survey completion took about 20 minutes. All study participants provided written informed consent.

\section{Institution and ethics approval and informed consent}

The study received ethical approval from the University of South Florida's Institutional Review Board (IRB No.: Pro00038628) and Moffitt Cancer Center Scientific Review Committee (IRB No.: MCC 20012).

Upon completion of the background survey, participants were provided detailed instructions for smartphone-based EMA that incorporated a specific mobile app. The EMA included 4 prompts (upon-waking, before-lunch, afternoon, and before-bedtime) per day for $14 \mathrm{~d}$. The uponwaking prompt included questions about the previous night's sleep. The other three prompts included questions regarding sleepiness and other daily experiences. Re- sponding to questions in each prompt took $5 \mathrm{~min}$ or less. All participants received check-in calls from research assistants after 2 nd and 7 th $\mathrm{d}$ of their study to encourage compliance and address questions.

During the same 14-d period as the EMA, participants were asked to wear an Actiwatch Spectrum plus device (Philips-Respironics, Murrysville, PA, USA) which measures wrist movement to quantify sleep and wake patterns. Of the 62 background survey participants, 61 participated in the actigraphy study. Using Actiware v6.09 software (Philips-Respironics, Murrysville, PA, USA), data were computer-scored using a previously-validated algorithm ${ }^{26,27)}$. A trained actigraphy scoring team determined the validity of each recording based on study-specific sleep criteria informed by previous literature on nurses' sleep health ${ }^{28)}$. Any intervals with a $15+$ minute discrepancy between scorers for total time in bed or sleep timing were reviewed by the senior investigator until agreement. Scoring was adjudicated by consensus.

At the end of the study, participants were compensated for their participation and time with a \$30 Amazon gift card. Participants who responded to more than $80 \%$ of EMA prompts received an additional \$20 Amazon gift card. Thus, the maximum compensation that participants could receive was $\$ 50$. All procedures were approved by the University of South Florida Institutional Review Board and Moffitt Cancer Center Scientific Review Committee.

\section{Background survey measures}

Sociodemographic and work characteristics. Participants reported their age, sex, race/ethnicity, the highest level of education, annual household income, current marital status, and the number of children living in the household. Participants also provided information about their weekly work hours, tenure with the current hospital and total tenure as a nurse, work schedule, and secondary workplaces.

Perceived stress. To assess participants' overall stress levels, we used the widely-used and well-validated Perceived Stress Scale (PSS) ${ }^{29)}$. We used the Short Form that is found to have acceptable psychometric properties ${ }^{30)}$. The PSS- 4 includes four items that measure the extent to which participants perceive their lives as unpredictable, uncontrollable, and overloaded. The instrument asks respondents to rate how often they experienced stressful situations in the past $30 \mathrm{~d}$ on a Likert scale ranging from 1 to 5 where $1=$ never and $5=$ very often. Example items read, "(During the past $30 \mathrm{~d}$, how often have you felt:) Difficulties were piling up so high that you could not over- 
come them?, Confident about your ability to handle your personal problems?" The sum of four items was calculated after reverse scoring the appropriate items. Higher scores indicated higher levels of perceived stress with values $\geq 8$ (no/little stress) suggesting an elevated level of stress ${ }^{31}$. The Cronbach alpha of the PSS-4 in this sample was 0.74.

Sleep concerns. To assess participants' main sleep concerns, we included an open-ended question worded "What is your main concern in your sleep that you want to improve?" Responses were coded as texts.

Interest and preferred form of a future sleep intervention were measured by three questions. The first question asked: "If a sleep-focused intervention is offered in the future (without any financial cost on your part), would you maybe be interested in participating or hearing more information?" Participants answered yes or no. Second, we asked, "For the sleep-focused intervention, which form of delivery would you prefer?" Participants were instructed to select all that apply to them of the following responses: group meetings at the workplace, one-on-one meetings at the workplace, online, or other (specify). Finally, we asked, "If you were to take part in a future sleep-focused intervention, which of the following would you like to participate in?" Again, participants selected all responses that applied to them: sleep hygiene education, mindfulnessbased stress reduction, cognitive-behavioral therapy for insomnia, or other (specify).

\section{EMA measures}

EMA measures included workday (vs. non-workday) and the following five EMA sleep measures-sleep sufficiency, sleep quality, sleep onset latency, nocturnal or early awakenings, and daytime sleepiness. Type of day was determined by asking during the before-bedtime prompt, "Was today a work day?" Responses were coded as yes (=workday) or no (=non-workday). The five sleep measures were adapted from previous studies ${ }^{24,32)}$. Except daytime sleepiness, all the items were asked during the upon-waking prompt, which was participant-initiated to avoid interrupting participants' sleep.

Sleep sufficiency was measured by asking, "Did you feel rested upon waking this morning?" on a scale of 0 (not at all) to 3 (extremely). Sleep quality was measured by asking, "How would you rate your sleep quality overall last night?" on a scale of 0 (very bad) to 3 (very good). Sleep onset latency was measured by asking, "How long did it take to fall asleep last night?". Responses were coded in minutes. Nocturnal or early awakenings were measured by asking, "Did you wake up in the middle of the night or early in the morning?". Participants responded yes or no. Daytime sleepiness was measured three times per day (before-lunch, afternoon, and before-bedtime) by asking, "How likely are you to doze off or fall asleep right now, in contrast to feeling just tired?" Responses were coded as 0 (not at all) to 3 (extremely). Scores were averaged across times within each day to create daily mean of sleepiness.

\section{Actigraphy measures}

We selected four actigraphy sleep measures that encompass the duration and disturbance of sleep. Those are main sleep duration, total time in bed, wake after sleep onset (WASO), and nap duration. The cut-point of each day, which is the time at which a $24-\mathrm{h}$ period begins and ends, was determined individually for each participant as the clock time closest to 12:00 pm that (a) does not cut through a main sleep period, (b) maximizes the number of valid days, (c) intersects the fewest number of naps, and (d) intersects the fewest number of off-wrist periods.

Main sleep duration was calculated by subtracting wake after sleep onset (WASO) from total time spent in bed. Total time in bed was calculated as the difference between sleep onset and sleep offset during a 24-h cut-pointdetermined period. WASO was calculated as the sum of all activity minutes between sleep onset and sleep offset each day. Examples of WASO include tossing and turning or using the restroom. Nap duration was calculated as the daily mean time (in minutes) across nap episodes within a day.

\section{Analytic strategy}

We first used descriptive statistics to examine sample characteristics. Next, we analyzed open-ended responses with a qualitative content analysis. To begin, the study team identified key themes/textual units that emerged across multiple participants ${ }^{33)}$. Next, three trained scorers independently categorized each response into primary and secondary themes from the list of themes provided. Categorization of responses followed an iterative process, such that themes were modified if individual scorers identified potential room for improvement. The study team adjudicated categorization discrepancies between individual scorers, considering primary and secondary themes identified by them. Following the iterative categorization process, responses were categorized into several common themes including insomnia-related themes. Descriptive statistics were used to summarize data. T-tests or $\chi^{2}$ tests were used to compare differences in sociodemographic and work characteristics as well as in perceived stress by the 
presence of insomnia-related concerns. Multilevel models in SAS 9.4 were used to compare nightly sleep characteristics by the presence of insomnia-related concerns (level-2 predictor), by type of day (level-1 predictor), or by the interaction between the two. PROC MIXED was used for continuous sleep variables and PROC GLIMMIX was used for nocturnal or early awakenings, which were a binary variable. These analyses were adequately powered based on previous research reporting that only a small sample size at level two ( $<50$ individuals) leads to biased estimates of the second-level standard errors in multilevel modeling. ${ }^{34)}$ Workday (vs. non-workday) was aligned with last night's sleep variables (except sleepiness variable that was measured during the day) to capture the potential effect of worry and stress about next workday on sleep, often observed in subjects with insomnia ${ }^{18,19)}$. To probe any significant interactions, we used estimate commands within multilevel models.

\section{Results}

\section{Sample characteristics}

Sociodemographic and work characteristics of the sample are shown in Table 1. Participants were on average $35 \mathrm{yr}$ old. The majority (92\%) were female; only 5 were male. Seventy-nine percent were non-Hispanic and 21\% were Hispanic. Sixty-six percent were White, $15 \%$ were Black or African American, 10\% were Asian, and the rest $(10 \%)$ were multi-racial or had some other race. The average level of education was close to 4 , which indicates college graduates. Mean annual household income range was approximately 4 , which indicates $\$ 80,000-\$ 99,999$. Forty-five percent were married, $13 \%$ were cohabitating or living with a permanent romantic partner, $13 \%$ were divorced, and $29 \%$ were never married. Sixty-one percent did not have a child; $18 \%$ had one child and $21 \%$ had two or more children. Our sample of nurses was approximately 10 yr younger than registered nurses in the U.S., but generally similar in other characteristics including gender, race/ethnicity, and education ${ }^{35}$.

Participants worked $37 \mathrm{~h}$ per week at the hospital on average. The average tenure with the hospital was $5.35 \mathrm{yr}$ and the average tenure as a nurse was 8.28 yr. Sixty-three percent worked regular day shift (7 am-7 pm) and 35\% worked regular night shift (7 pm-7 am). One nurse worked other shift, which was a fixed $11 \mathrm{am}-11 \mathrm{pm}$ schedule. The majority $(90 \%)$ did not have a second job outside of the hospital where data were collected. The average level of perceived stress exceeded $8(M=9.55)$, meaning that our sample had an elevated level of stress overall.

\section{Qualitative themes on main sleep cncerns}

Figure 1 shows a flowchart of nurses' responses to the question asking main sleep concerns and examples of the text narratives and how we categorized each narrative. Out of 62 participants, 60 provided open-ended responses to the question asking main concerns in their sleep. Among those who responded, 3 participants indicated no concern and 57 reported having any sleep concern. Participants' responses on sleep concerns were categorized into the following five themes: difficulty initiating and/or maintaining asleep $(n=39)$, sleep schedule $(n=6)$, insufficient amount of sleep $(n=5)$, non-restorative sleep $(n=3)$, and other $(n=4)$. Thus, insomnia-related concerns that comprise of difficulty initiating and/or maintaining asleep and non-restorative sleep were most prevalent with $68 \%(n=42$ out of 62$)$ indicating as their main sleep concerns. Participants whose answers about sleep concerns were classified into either "difficulty initiating and/or maintaining asleep" or "nonrestorative sleep" comprised the group of participants "with insomnia concerns" used in further analyses.

As shown in Table 1, participants who indicated insomnia-related concerns $(n=42)$ did not significantly differ from those who did not indicate insomnia concerns $(n=20)$ in sociodemographic and work characteristics. The only difference was observed in perceived stress. Nurses with insomnia-related concerns reported significantly higher perceived stress compared to nurses without insomnia concerns. The difference between the groups corresponded to an approximately 2 -unit change in perceived stress.

\section{EMA and actigraphy sleep characteristics by the presence} of insomnia concerns and by type of day

Out of 62 participants, 61 provided the EMA and actigraphy sleep data, with average 15.93 valid EMA days and 13.62 actigraphy days per participant, well adhering to the 14-d study protocol. Forty-three percent of the days were workdays. Table 2 shows results of multilevel models comparing EMA and actigraphy sleep characteristics by the presence of insomnia concerns and by type of day. Beginning with differences in EMA sleep characteristics by insomnia concerns, participants with insomnia-related concerns reported lower sleep sufficiency and worse sleep quality compared to participants without insomnia concerns, on average across days. There were no statistically significant differences between the groups in sleep onset latency, nocturnal or early awakenings, and sleepiness. There were no significant differences in actigraphy sleep 
Table 1. Sociodemographic and work characteristics of the sample and differences by the presence of insomnia concerns

\begin{tabular}{|c|c|c|c|c|c|c|c|c|c|c|}
\hline & \multicolumn{3}{|c|}{$\begin{array}{c}\text { Overall sample } \\
\qquad(n=62)\end{array}$} & \multicolumn{3}{|c|}{$\begin{array}{l}\text { Those with insomnia- } \\
\text { related concerns } \\
(n=42)\end{array}$} & \multicolumn{3}{|c|}{$\begin{array}{l}\text { Those without insomnia } \\
\text { concerns } \\
(n=20)\end{array}$} & \multirow[t]{2}{*}{ Differencetest $^{4}$} \\
\hline & $n$ & $M$ or $\%$ & $(S D)$ & $n$ & $M$ or $\%$ & $(S D)$ & $n$ & $M$ or $\%$ & $(S D)$ & \\
\hline \multicolumn{11}{|c|}{ Sociodemographic characteristics } \\
\hline Age (yr) & 62 & 35.26 & $(11.69)$ & 42 & 36.26 & $(12.02)$ & 20 & 33.15 & $(10.95)$ & -0.98 \\
\hline Sex & 62 & & & 42 & & & 20 & & & 0.37 \\
\hline Female & 57 & $92 \%$ & & 38 & $90 \%$ & & 19 & $95 \%$ & & \\
\hline Male & 5 & $8 \%$ & & 4 & $10 \%$ & & 1 & $5 \%$ & & \\
\hline Hispanic & 62 & & & 42 & & & 20 & & & 0.63 \\
\hline No & 49 & $79 \%$ & & 32 & $76 \%$ & & 17 & $85 \%$ & & \\
\hline Yes & 13 & $21 \%$ & & 10 & $24 \%$ & & 3 & $15 \%$ & & \\
\hline Race & 61 & & & 41 & & & 20 & & & 4.63 \\
\hline White & 40 & $65 \%$ & & 30 & $73 \%$ & & 10 & $50 \%$ & & \\
\hline Black or African American & 9 & $15 \%$ & & 4 & $10 \%$ & & 5 & $35 \%$ & & \\
\hline Asian & 6 & $10 \%$ & & 3 & $7 \%$ & & 3 & $15 \%$ & & \\
\hline Other & 6 & $10 \%$ & & 4 & $10 \%$ & & 2 & $10 \%$ & & \\
\hline Education ${ }^{1}$ & 62 & 4.06 & $(0.72)$ & 42 & 4.02 & $(0.72)$ & 20 & 4.15 & $(0.75)$ & 0.64 \\
\hline Household annual income ${ }^{2}$ & 62 & 3.94 & $(2.17)$ & 42 & 4.05 & $(2.25)$ & 20 & 3.7 & $(2.03)$ & -0.59 \\
\hline Marital status & 62 & & & 42 & & & 20 & & & 0.75 \\
\hline Married & 28 & $45 \%$ & & 19 & $45 \%$ & & 9 & $45 \%$ & & \\
\hline Cohabitating & 8 & $13 \%$ & & 6 & $14 \%$ & & 2 & $10 \%$ & & \\
\hline Divorced & 8 & $13 \%$ & & 6 & $14 \%$ & & 2 & $10 \%$ & & \\
\hline Never married & 18 & $29 \%$ & & 11 & $26 \%$ & & 7 & $35 \%$ & & \\
\hline Number of children & 62 & & & 42 & & & 20 & & & 0.51 \\
\hline No child & 38 & $61 \%$ & & 26 & $62 \%$ & & 12 & $60 \%$ & & \\
\hline One child & 11 & $18 \%$ & & 7 & $17 \%$ & & 4 & $20 \%$ & & \\
\hline Two or more children & 13 & $21 \%$ & & 9 & $21 \%$ & & 4 & $20 \%$ & & \\
\hline \multicolumn{11}{|l|}{ Work characteristics } \\
\hline Work hours (h/wk) & 62 & 36.78 & $(4.30)$ & 42 & 36.62 & $(4.73)$ & 20 & 37.13 & $(3.29)$ & 0.43 \\
\hline Company tenure (yr) & 61 & 5.35 & $(6.16)$ & 42 & 5.97 & $(6.77)$ & 19 & 3.96 & $(4.40)$ & -1.18 \\
\hline Nurse tenure (yr) & 61 & 8.28 & $(8.78)$ & 42 & 8.96 & $(9.70)$ & 19 & 6.79 & $(6.24)$ & -1.05 \\
\hline Work schedule & 62 & & & 42 & & & 20 & & & 2.56 \\
\hline Day shift & 39 & $63 \%$ & & 28 & $67 \%$ & & 11 & $55 \%$ & & \\
\hline Night shift & 22 & $35 \%$ & & 14 & $33 \%$ & & 8 & $40 \%$ & & \\
\hline Other & 1 & $2 \%$ & & & & & 1 & $5 \%$ & & \\
\hline Having second job & 62 & & & 42 & & & 20 & & & 0.74 \\
\hline No & 56 & $90 \%$ & & 37 & $88 \%$ & & 19 & $95 \%$ & & \\
\hline Yes & 6 & $10 \%$ & & 5 & $12 \%$ & & 1 & $5 \%$ & & \\
\hline \multicolumn{11}{|l|}{ Overall Stress } \\
\hline Perceived stress scale (4-20) & 62 & 9.55 & $(2.80)$ & 42 & 10.21 & $(2.81)$ & 20 & 8.15 & $(2.28)$ & $-2.87^{* *}$ \\
\hline
\end{tabular}

${ }^{1}$ Education was coded as 1 (Grade 12 or GED) to 6 (postgraduate degree or related credential); the sample average was close to 4 , which indicates college graduates. ${ }^{2}$ Household annual income was coded as 1 (less than 40,000) to 10 (more than 200,000); the sample average was close to 4, which indicates $80,000-99,999 .{ }^{3}$ Participants who indicated "Difficulty falling and/or staying asleep $(n=39)$ " or "Not feeling rested upon waking ( $\left.\mathrm{n}=3\right)$ " as their main sleep concerns. ${ }^{4} \mathrm{~T}$-tests (for continuous variables) or $\chi^{2}$ tests (for binary variables) were used.

$* * p<0.01$. 


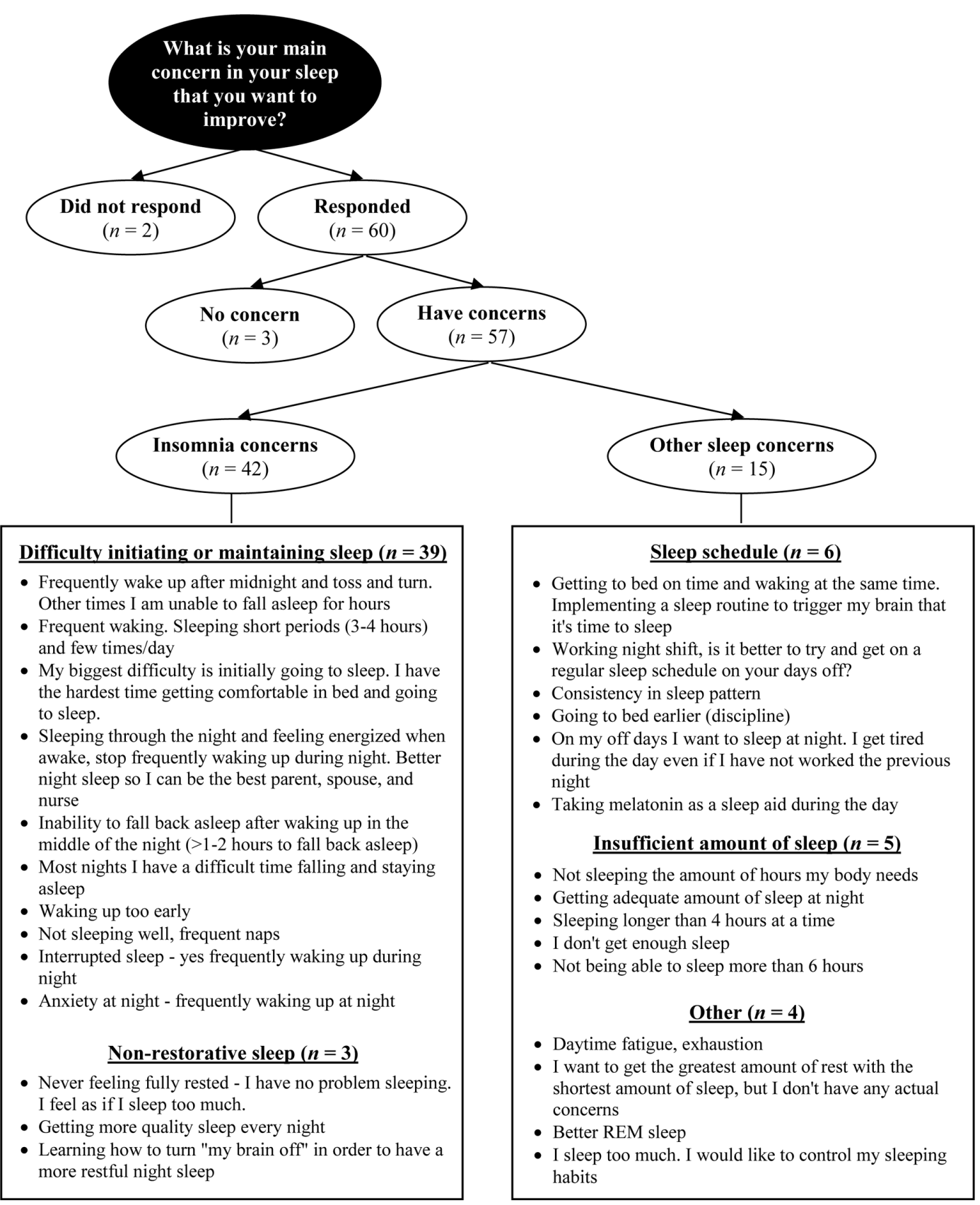

Bullet points are participants' own narratives. For "Difficulty initiating or maintaining sleep", only part of participant narratives is provided due to lack of space.

Fig. 1. Flowchart of participant responses to the open-ended question on main sleepconcerns, qualitative themes, and participant narratives.

variables. In terms of differences in EMA sleep characteristics between workdays and non-workdays, participants reported lower sleep sufficiency, worse sleep quality, and more sleepiness (but not in sleep onset latency or nocturnal or early awakenings) on nights before workdays compared to on nights before non-workdays. There were also differences between workdays and non-workdays in all actigraphy sleep characteristics, such that participants had shorter main sleep duration, shorter total time in bed ( $B=-1.64, S E=0.14, p<0.001)$, less WASO, and shorter nap duration on nights before workdays compared to on nights before non-workdays. Figure 2 visually depicts these estimated differences.

There was a significant interaction between the presence of insomnia concerns and type of day in actigraphy nap duration ( $B=23.97, S E=11.86, p<0.05)$. The post-hoc 
Table 2. Results of multilevel models examining differences in sleep characteristics by the presence of insomnia concerns and by type of day

\begin{tabular}{|c|c|c|c|c|c|c|c|c|}
\hline & \multicolumn{8}{|c|}{ EMA $^{3}$ sleep } \\
\hline & \multicolumn{2}{|c|}{$\begin{array}{l}\text { Sleep sufficiency } \\
\text { ( } 0 \text { to } 3=\text { higher })\end{array}$} & \multicolumn{2}{|c|}{$\begin{array}{l}\text { Sleep quality } \\
\text { (0 to } 3=\text { better) }\end{array}$} & \multicolumn{2}{|c|}{$\begin{array}{l}\text { Sleep onset latency } \\
\text { (in minutes) }\end{array}$} & \multicolumn{2}{|c|}{$\begin{array}{l}\text { Nocturnal or early } \\
\text { awakenings } \\
\text { (yes vs. no) }\end{array}$} \\
\hline & $B$ & $(S E)$ & $B$ & $(S E)$ & $B$ & $(S E)$ & $B$ & $(S E)$ \\
\hline \multicolumn{9}{|l|}{ Fixed Effects } \\
\hline Intercept & $1.87 * * *$ & $(0.10)$ & $2.05 * * *$ & $(0.08)$ & $19.08 * * *$ & $(3.86)$ & 0.48 & $(0.38)$ \\
\hline Those with Insomnia Concerns vs. Those without ${ }^{1}$ & $-0.30 *$ & $(0.12)$ & $-0.23 *$ & (0.10) & 4.88 & $(4.61)$ & 0.68 & $(0.46)$ \\
\hline Workdays vs. Non-workdays ${ }^{2}$ & $-0.34 * * *$ & $(0.06)$ & $-0.21 * * *$ & $(0.06)$ & 0.75 & $(1.82)$ & 0.12 & $(0.18)$ \\
\hline \multicolumn{9}{|l|}{ Random Effects } \\
\hline Person level variance & $0.12 * * *$ & $(0.04)$ & $0.07 * * *$ & $(0.02)$ & $212.96^{* * *}$ & $(50.19)$ & $2.07 * * *$ & $(0.56)$ \\
\hline \multirow[t]{4}{*}{ Residual variance } & $0.57 * * *$ & $(0.03)$ & $0.45^{* * *}$ & $(0.03)$ & $436.21 * * *$ & $(26.84)$ & $0.79 * * *$ & $(0.05)$ \\
\hline & \multicolumn{2}{|c|}{ EMA sleep } & \multicolumn{6}{|c|}{ Actigraphy sleep } \\
\hline & \multicolumn{2}{|c|}{$\begin{array}{c}\text { Sleepiness } \\
\text { (0 to } 3=\text { extremely) }\end{array}$} & \multicolumn{2}{|c|}{$\begin{array}{l}\text { Sleep duration } \\
\text { (in minutes) }\end{array}$} & \multicolumn{2}{|c|}{$\begin{array}{c}\mathrm{WASO}^{6} \\
\text { (in minutes) }\end{array}$} & \multicolumn{2}{|c|}{$\begin{array}{l}\text { Nap duration } \\
\text { (in minutes) }\end{array}$} \\
\hline & $B$ & $(S E)$ & $B$ & $(S E)$ & $B$ & $(S E)$ & $B$ & $(S E)$ \\
\hline \multicolumn{9}{|l|}{ Fixed Effects } \\
\hline Intercept & $0.84 * * *$ & $(0.09)$ & $7.5^{* * *}$ & $(0.22)$ & $38.83^{* * *}$ & $(3.83)$ & $52.27 * * *$ & $(8.23)$ \\
\hline Those with Insomnia Concerns vs. Those without ${ }^{1}$ & -0.06 & $(0.10)$ & 0.09 & $(0.27)$ & 7.39 & $(4.65)$ & -15.54 & $(9.83)$ \\
\hline Workdays vs. Non-workdays ${ }^{2}$ & $0.13 * *$ & $(0.04)$ & $-1.42 * * *$ & $(0.13)$ & $-11.93 * * *$ & (1.50) & $-24.68 * * *$ & (5.67) \\
\hline \multicolumn{9}{|l|}{ Random Effects } \\
\hline Person level variance & $0.12 * * *$ & $(0.03)$ & $0.71 * * *$ & $(0.18)$ & $249.87 * * *$ & $(52.75)$ & $802.44 * * *$ & $(252.2)$ \\
\hline Residual variance & $0.31 * * *$ & $(0.02)$ & $2.39 * * *$ & $(0.14)$ & $334.88 * * *$ & $(19.29)$ & $4903.42 * * *$ & $(283.5)$ \\
\hline
\end{tabular}

${ }^{1}$ Participants who indicated "Difficulty falling and/or staying asleep $(n=39)$ " or "Not feeling rested upon waking $(\mathrm{n}=3)$ " as their primary sleep concerns. ${ }^{2}$ Workday (vs. non-workday) was aligned with last night's sleep variables, except sleepiness measured during the day. ${ }^{3}$ EMA: Ecological momentary assessment. ${ }^{4}$ Nocturnal or Early Awakenings were modeled using PROC GLIMMIX for a binary outcome. ${ }^{5}$ Results on total time in bed were consistent $(B$ $=-1.64, S E=0.14, p<0.001) .{ }^{6}$ WASO: Wake After Sleep Onset.

$* p<0.05, * * p<0.01, * * * p<0.001$.

simple slopes test (see Fig. 2) revealed that both groups of participants (those with insomnia concerns and those without) had shorter actigraphy nap duration prior to workdays than prior to non-workdays. However, participants without insomnia concerns had longer nap duration prior to non-workdays, contributing to a larger difference between workdays and non-workdays (Slope Estimate $=-40.23, S E=9.55, p<0.001)$ than that for participants with insomnia-related (Slope Estimate $=-16.26, S E=7.02$, $p<0.05)$. There were no significant interactions between the presence of insomnia concerns and type of day in other sleep variables.

\section{Interest and preferred form of a future sleep intervention}

Nearly all nurses $(n=59,95 \%)$ expressed interest in participating in a future sleep intervention. Figure 3 shows preferred delivery formats and contents assessed by these nurses. An online delivery format was most preferred (58\%), followed by group meetings at the workplace (53\%) and one-on-one meetings at the workplace $(27 \%)$. With re- gards to intervention contents, mindfulness strategies were endorsed by most nurses (73\%), followed by cognitivebehavioral therapy for insomnia (49\%), and sleep hygiene education (36\%). Other responses indicated that they would be interested in participating in "any" forms of a sleep intervention. Many nurses (35\%) selected more than one option.

\section{Supplementary analyses}

We further examined differences by work shift. There were no differences between day-shift nurses (including one with 11 am-11 pm schedule) and night-shift nurses in sleep concerns, the prevalence of insomnia-related concerns, or interest in a sleep intervention. There were no differences in preferences for intervention contents. Regarding delivery formats, an online format was more preferred by night-shift workers (68\%) than by day-shift nurses $(50 \%)$; group meetings were more preferred by day-shift workers (53\%) than by night-shift nurses (45\%). 

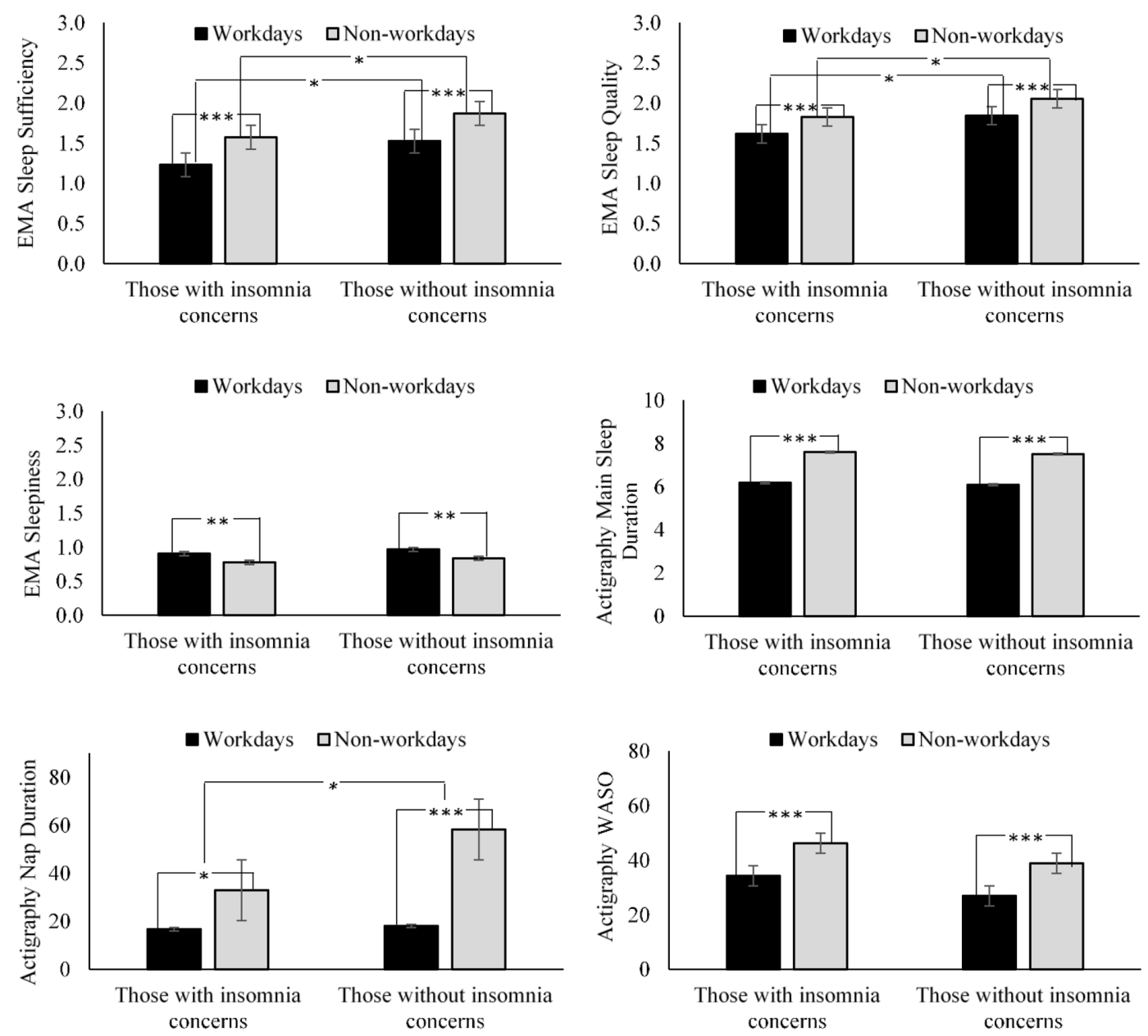

Fig. 2. EMA and actigraphy sleep characteristics by the presence of insomnia concerns and by type of day.

Workday (vs. non-workday) was aligned with last night's sleep variables, except sleepiness that was measured during the day. EMA: Ecological momentary assessment; WASO: Wake After Sleep Onset. Only statistically significant differences are indicated. ${ }^{*} p<0.05,{ }^{* *} p<0.01, * * * p<0.001$.
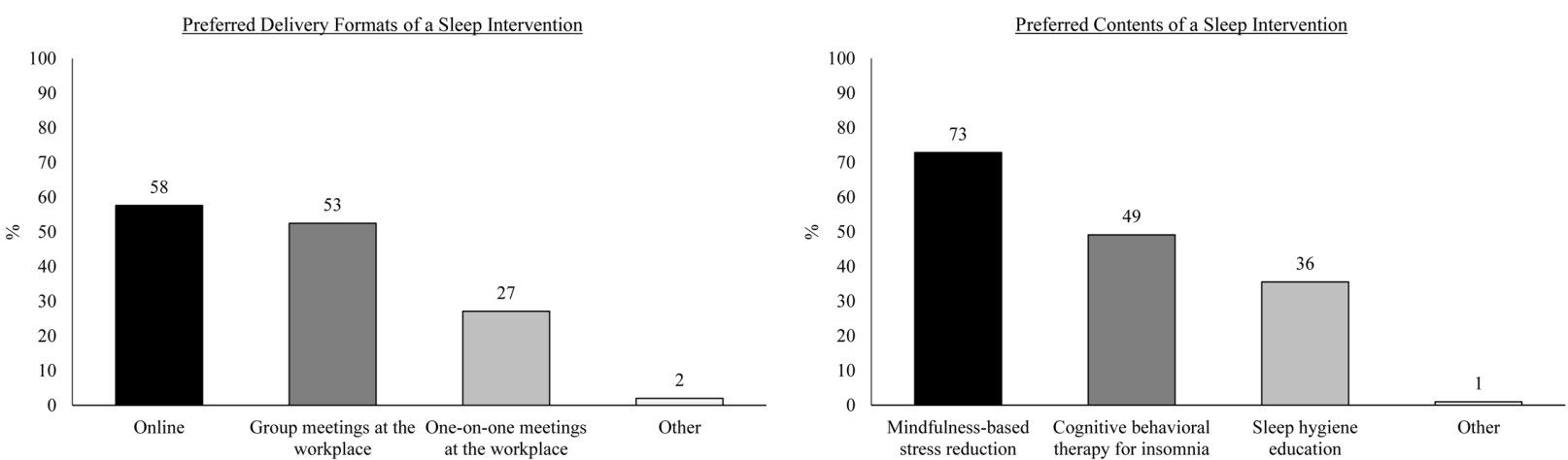

Fig. 3. Preferred delivery formats and contents of a sleep intervention.

Percentages were calculated among a sub-group of participants who expressed interest in participating in a future sleep intervention $(n=59$, out of $N=62$ ). Participants were instructed to select all that apply to them among the given options. Other responses indicated "any". 


\section{Discussion}

The current study yields several key findings that advance our understanding on insomnia symptoms and overall sleep issues in healthcare workers. We found a high prevalence of insomnia-related concerns in a sample of U.S. hospital nurses. Compared to those without insomnia concerns, those with insomnia-related concerns reported significantly higher perceived stress and had lower sleep sufficiency and sleep quality measured by 14-d ecological momentary assessment (EMA). Participants with insomnia concerns also had shorter actigraphy-measured nap duration prior to non-workdays than those without. Moreover, we found overwhelmingly high interest and willingness to participate in a sleep intervention in this sample. Although some sources of sleep concerns may not be directly intervenable (e.g., sleep schedule due to working at night), a well-designed sleep intervention may better equip nurses for sufficient and good quality sleep during the time that they do have. Below, we discuss implications of our key findings.

The high prevalence of insomnia-related concerns in this sample of nurses raises a potential issue regarding the adverse impact of poor sleep on the health and well-being of these nurses ${ }^{36-38)}$ as well as the quality of patient care delivery ${ }^{6-8,24)}$. Although we expected a high prevalence of insomnia-related concerns in nurses, the estimated prevalence in this sample $(68 \%)$ was much higher than expected. Note that we used a wider criterion than the ones used in previous studies ${ }^{7,12)}$. Not all of the nurses who indicated insomnia-related concerns may have a clinical insomnia disorder based on the DSM-5 criteria $^{11)}$; however, having concerns regarding insomnia may have negative impact on nurses' daily functioning on its own. More data are needed to estimate a prevalence of insomnia-related concerns in a larger sample of nurses across diverse settings.

Insomnia-related concerns were further validated against multiple sleep characteristics assessed by 14-d EMA and actigraphy. Nurses who reported insomnia-related concerns also reported lower sleep sufficiency and worse sleep quality overall across EMA days than those without insomnia concerns. Moreover, significant workday effect was found; nurses had poorer sleep characteristics prior to workdays, potentially due to work-related anticipatory stress and worry ${ }^{18,19,39)}$. That is, independent of insomniarelated concerns, nurses had lower sleep sufficiency, worse sleep quality, more sleepiness, shorter main sleep duration, shorter total time in bed, less WASO (potentially due to shorter total time in bed), and shorter nap duration prior to workdays than prior to non-workdays. However, such workday effect was more apparent for nurses with insomnia-related concerns in terms of nap duration. Prior to nonworkdays, nurses without insomnia concerns increased nap duration as much as 40 minutes, whereas nurses with insomnia concerns increased nap duration as much as 16 min. This 24-min gap per day in napping can have a meaningful difference in respite and daily functioning overall ${ }^{40,41)}$. Note that none of the groups had an extensive napping ( $\geq 60 \mathrm{~min} / \mathrm{d}$ ). The interaction result on nap duration may suggest that those with potential insomnia still cannot compensate insufficient sleep when they are free from work-related anticipatory stress and worry. Furthermore, there were dose-response relationships such that sleep sufficiency, sleep quality, and nap duration were lowest for nurses with insomnia-related concerns before workdays and highest for nurses without insomnia concerns before non-workdays. Previous studies reported that those with insomnia experience poor sleep quality or disturbed sleep in general ${ }^{13-16)}$. The current study contributes to the occupational health literature by showing that nurses' sleep characteristics prior to workdays are poorer and this is more apparent for those with insomnia symptoms.

Our findings show that future studies should focus more on improving sleep in nurses. The vast majority of our sample $(92 \%)$ reported having at least one concern in their sleep. Moreover, nearly all participants $(95 \%)$ reported that they are interested in participating in a sleep intervention if offered in the future. These results indicate that nurses clearly recognize issues in their sleep and want to do something to resolve the issues, which may reinforce their motivation to follow suggested regimen in future intervention studies ${ }^{42)}$. Moreover, this sample provided preferred formats and contents for a future sleep intervention. As an intervention customized for a target group is found to be more effective ${ }^{25}$, data collected from this study could guide the design of a future sleep intervention to offer the most appealing and approachable form to nurses.

Based on our data, an online sleep intervention that includes mindfulness strategies may be effective to improve insomnia symptoms and overall sleep health in nurses. Online interventions have several advantages over traditional in-person interventions, such as their ability to deliver treatment in a more time- and cost-effective manner, customize treatment protocols by participant needs, and monitor participant compliance ${ }^{43,44)}$. It could also include group discussions via online, as group meetings were also preferred by nurses but arranging physical group meetings at the workplace may be challenging due to different work schedules 
and sometimes it may not be even feasible especially during global pandemic outbreaks. The mindfulness-based stress reduction program was most preferred by nurses and found to be effective to improve sleep in other populations like breast cancer survivors ${ }^{45}$. As many nurses selected more than one option, a multicomponent online sleep intervention could be used to serve diverse needs of nurses.

\section{Limitations and future directions}

This study has several strengths, including the use of various sources of data (i.e., survey, EMA, and actigraphy), mixed methods (i.e., qualitative and quantitative), and comparison of insomnia-specific sleep characteristics on workdays vs. on non-workdays. There are, of course, also limitations of this study. The primary limitation of this study is a small sample size recruited from one hospital. It is possible that nurses who had sleep problems would have been more willing to participate in the study and this might have been reflected in the high interest in a sleep intervention. Future research could consider collecting data across multiple hospitals by using a random recruitment strategy to increase generalizability of findings. Another potential limitation is our inability to determine whether the reported insomnia-related concerns meet criteria for insomnia disorder. One of the DSM-5 criteria to determine insomnia disorder is that a sleep difficulty is present for at least 3 months occurring 3 or more times per week and causes significant daytime impairment ${ }^{11)}$. In this study, we used an open-ended question to ask participants about their main sleep concern that they want to improve, and by doing so, we were unable to specify the length or frequency of insomnia symptoms and associated daytime impairment. To increase precision in potential insomnia detection, future studies need to assess the insomnia disorder diagnostic criteria and rule out other sleep disorders. Lastly, the aim of this study was to describe sleep issues in healthcare workers. Based on our findings, future studies could examine how sleep characteristics in healthcare workers are related to fatigue and other daily experiences. Despite these limitations, our study is unique in documenting insomnia-related concerns in nurses and corresponding insomnia-specific sleep characteristics assessed by 14-d EMA and actigraphy, which adds new knowledge to the literature and brings scholarly attention to this underserved group.

\section{Conclusion}

The current study finds that insomnia-related concerns are prevalent in a sample of U.S. hospital nurses, and their concerns are supported by subjective and objective measures of daily sleep. The information obtained from this study could be used as the basis for developing an intervention to improve insomnia and overall sleep health in nurses, and more broadly healthcare workers. Importantly, improvement in healthcare workers' insomnia symptoms has potential to improve the health of both workers and patients.

\section{Authors' Contributions}

S. Lee initiated the study, conducted analyses, and drafted the manuscript. B. D. Gonzalez and B. Small contributed to the design and data collection of the parent study. All authors contributed to the revision of this manuscript and approved the final submitted version.

\section{Funding}

This work was supported, in part, by the University of South Florida College of Behavioral \& Community Sciences Internal Grant Program (PI: Lee, Grant No. 0134930).

\section{Conflict of Interest}

None declared.

\section{Acknowledgement}

The authors thank Taylor Vigoureux, M.A., for her help with data and variable construction.

\section{References}

1) Cooley ME, Sipples RL, Murphy M, Sarna L (2008) Smoking cessation and lung cancer: oncology nurses can make a difference. Semin Oncol Nurs 24, 16-26. [Medline] [CrossRef]

2) Piper BF, Borneman T, Sun VCY, Koczywas M, Uman G, Ferrell B, James RL (2008) Cancer-related fatigue: role of oncology nurses in translating National Comprehensive Cancer Network assessment guidelines into practice. Clin J Oncol Nurs 12 Suppl, 37-47. [Medline] [CrossRef]

3) Atwal A, Tattersall K, Caldwell K, Craik C (2006) Multidisciplinary perceptions of the role of nurses and healthcare assistants in rehabilitation of older adults in acute health care. J Clin Nurs 15, 1418-25. [Medline] [CrossRef] 
4) Stringer B, Van Meijel B, De Vree W, Van der Bijl J (2008) User involvement in mental health care: the role of nurses. A literature review. J Psychiatr Ment Health Nurs 15, 678-83. [Medline] [CrossRef]

5) Armon G, Shirom A, Shapira I, Melamed S (2008) On the nature of burnout-insomnia relationships: a prospective study of employed adults. J Psychosom Res 65, 5-12. [Medline] [CrossRef]

6) Kousloglou S, Mouzas O, Bonotis K, Roupa Z, Vasilopoulos A, Angelopoulos N (2014) Insomnia and burnout in Greek Nurses. Hippokratia 18, 150-5. [Medline]

7) Swanson LM, Arnedt JT, Rosekind MR, Belenky G, Balkin TJ, Drake C (2011) Sleep disorders and work performance: findings from the 2008 National Sleep Foundation Sleep in America poll. J Sleep Res 20, 487-94. [Medline] [CrossRef]

8) Lockley SW, Cronin JW, Evans EE, Cade BE, Lee CJ, Landrigan CP, Rothschild JM, Katz JT, Lilly CM, Stone PH, Aeschbach D Czeisler CA, Harvard Work Hours, Health and Safety Group (2004) Effect of reducing interns' weekly work hours on sleep and attentional failures. N Engl J Med 351, 1829-37. [Medline] [CrossRef]

9) Ohayon MM (2002) Epidemiology of insomnia: what we know and what we still need to learn. Sleep Med Rev 6, 97-111. [Medline] [CrossRef]

10) Kronholm E, Partonen T, Laatikainen T, Peltonen M, Härmä M, Hublin C, Kaprio J, Aro AR, Partinen M, Fogelholm M, Valve R, Vahtera J, Oksanen T, Kivimäki M, Koskenvuo M, Sutela H (2008) Trends in self-reported sleep duration and insomnia-related symptoms in Finland from 1972 to 2005: a comparative review and re-analysis of Finnish population samples. J Sleep Res 17, 54-62. [Medline] [CrossRef]

11) American Psychiatric Association (2013) Diagnostic and Statistical Manual of Mental Disorders, 5th Ed., American Psychiatric Association, Arlington.

12) Eldevik MF, Flo E, Moen BE, Pallesen S, Bjorvatn B (2013) Insomnia, excessive sleepiness, excessive fatigue, anxiety, depression and shift work disorder in nurses having less than 11 hours in-between shifts. PLoS One 8, e70882. [Medline] [CrossRef]

13) Drake CL, Roehrs T, Roth $T$ (2003) Insomnia causes, consequences, and therapeutics: an overview. Depress Anxiety 18, 163-76. [Medline] [CrossRef]

14) Shekleton JA, Rogers NL, Rajaratnam SMW (2010) Searching for the daytime impairments of primary insomnia. Sleep Med Rev 14, 47-60. [Medline] [CrossRef]

15) Ohayon MM, Roth $\mathrm{T}$ (2001) What are the contributing factors for insomnia in the general population? J Psychosom Res 51, 745-55. [Medline] [CrossRef]

16) Lichstein KL, Durrence HH, Taylor DJ, Bush AJ, Riedel BW (2003) Quantitative criteria for insomnia. Behav Res Ther 41, 427-45. [Medline] [CrossRef]

17) Morin CM, Rodrigue S, Ivers H (2003) Role of stress, arousal, and coping skills in primary insomnia. Psychosom Med 65, 259-67. [Medline] [CrossRef]

18) Carney CE, Waters WF (2006) Effects of a structured problem-solving procedure on pre-sleep cognitive arousal in college students with insomnia. Behav Sleep Med 4, 13-28. [Medline] [CrossRef]

19) Âkerstedt $T$ (2006) Psychosocial stress and impaired sleep. Scand J Work Environ Health 32, 493-501. [Medline] [CrossRef]

20) Marino M, Killerby M, Lee S, Klein LC, Moen P, Olson R, Kossek EE, King R, Erickson L, Berkman LF, Buxton OM (2016) The effects of a cluster randomized controlled workplace intervention on sleep and work-family conflict outcomes in an extended care setting. Sleep Health 2, 297-308. [Medline] [CrossRef]

21) Gordon AM, Chen S (2013) The role of sleep in interpersonal conflict: do sleepless nights mean worse fights? Soc Psychol Personal Sci 5, 168-75. [CrossRef]

22) Lee S, Crain TL, McHale SM, Almeida DM, Buxton OM, Buxton OM (2017) Daily antecedents and consequences of nightly sleep. J Sleep Res 26, 498-509. [Medline] [CrossRef]

23) Sin NL, Almeida DM, Crain TL, Kossek EE, Berkman LF, Buxton OM (2017) Bidirectional, temporal associations of sleep with positive events, affect, and stressors in daily life across a week. Ann Behav Med 51, 402-15. [Medline] [CrossRef]

24) Lee S, Buxton OM, Andel R, Almeida DM (2019) Bidirectional associations of sleep with cognitive interference in employees' work days. Sleep Health 5, 298-308. [Medline] [CrossRef]

25) Flink IK, Sfyrkou C, Persson B (2016) Customized CBT via internet for adolescents with pain and emotional distress: a pilot study. Internet Interv 4, 43-50. [Medline] [CrossRef]

26) Buxton OM, Lee S, Marino M, Beverly C, Almeida DM, Berkman L (2018) Sleep health and predicted cardiometabolic risk scores in employed adults from two industries. J Clin Sleep Med 14, 371-83. [Medline] [CrossRef]

27) Marino M, Li Y, Rueschman MN, Winkelman JW, Ellenbogen JM, Solet JM, Dulin H, Berkman LF, Buxton OM (2013) Measuring sleep: accuracy, sensitivity, and specificity of wrist actigraphy compared to polysomnography. Sleep (Basel) 36, 1747-55. [Medline] [CrossRef]

28) Geiger-Brown J, Rogers VE, Trinkoff AM, Kane RL, Bausell RB, Scharf SM (2012) Sleep, sleepiness, fatigue, and performance of 12-hour-shift nurses. Chronobiol Int 29, 211-9. [Medline] [CrossRef]

29) Cohen S, Kamarck T, Mermelstein R (1983) A global measure of perceived stress. J Health Soc Behav 24, 385-96. [Medline] [CrossRef]

30) Warttig SL, Forshaw MJ, South J, White AK (2013) New, normative, English-sample data for the Short Form Perceived Stress Scale (PSS-4). J Health Psychol 18, 1617-28. [Medline] [CrossRef]

31) Melnyk BM, Orsolini L, Tan A, Arslanian-Engoren C, 
Melkus GDE, Dunbar-Jacob J, Rice VH, Millan A, Dunbar SB, Braun LT, Wilbur J, Chyun DA, Gawlik K, Lewis LM (2018) A national study links nurses' physical and mental health to medical errors and perceived worksite wellness. J Occup Environ Med 60, 126-31. [Medline] [CrossRef]

32) Buysse DJ, Reynolds CF 3rd, Monk TH, Berman SR, Kupfer DJ (1989) The Pittsburgh Sleep Quality Index: a new instrument for psychiatric practice and research. Psychiatry Res 28, 193-213. [Medline] [CrossRef]

33) Charmaz K, Conducting grounded theory: a practical guide through qualitative analysis. SAGE Publications, 2006.

34) Maas CJ, Hox J (2005) Sufficient sample sizes for multilevel modeling. Methodology 1, 86-92. [CrossRef]

35) Health Resources and Services Adminstration (2013) The U.S. Nursing Workforce: Trends in Supply and Education.

36) Canivet C, Nilsson PM, Lindeberg SI, Karasek R, Östergren PO (2014) Insomnia increases risk for cardiovascular events in women and in men with low socioeconomic status: a longitudinal, register-based study. J Psychosom Res 76, 292-9. [Medline] [CrossRef]

37) Kalmbach DA, Pillai V, Drake CL (2018) Nocturnal insomnia symptoms and stress-induced cognitive intrusions in risk for depression: a 2-year prospective study. PLoS One 13, e0192088. [Medline] [CrossRef]

38) Vgontzas AN, Liao D, Pejovic S, Calhoun S, Karataraki M, Basta M, Fernández-Mendoza J, Bixler EO (2010) Insomnia with short sleep duration and mortality: the Penn State cohort. Sleep 33, 1159-64. [Medline] [CrossRef]

39) Jansson M, Linton SJ (2006) Psychosocial work stressors in the development and maintenance of insomnia: a prospective study. J Occup Health Psychol 11, 241-8.
[Medline] [CrossRef]

40) Hammouda O, Romdhani M, Chaabouni Y, Mahdouani K, Driss T, Souissi N (2018) Diurnal napping after partial sleep deprivation affected hematological and biochemical responses during repeated sprint. Biol Rhythm Res 49, 927-39.

41) Hsouna H, Boukhris O, Abdessalem R, Trabelsi K, Ammar A, Shephard RJ, Chtourou H (2019) Effect of different nap opportunity durations on short-term maximal performance, attention, feelings, muscle soreness, fatigue, stress and sleep. Physiol Behav 211, 112673. [Medline] [CrossRef]

42) Murphy MR, Escamilla MI, Blackwell PH, Lucke KT, Miner-Williams D, Shaw V, Lewis SL (2007) Assessment of caregivers' willingness to participate in an intervention research study. Res Nurs Health 30, 347-55. [Medline] [CrossRef]

43) Griffiths F, Lindenmeyer A, Powell J, Lowe P, Thorogood M (2006) Why are health care interventions delivered over the internet? A systematic review of the published literature. J Med Internet Res 8, e10. [Medline] [CrossRef]

44) Strecher V (2007) Internet methods for delivering behavioral and health-related interventions (eHealth). Annu Rev Clin Psychol 3, 53-76. [Medline] [CrossRef]

45) Lengacher CA, Reich RR, Paterson CL, Jim HS, Ramesar S, Alinat CB, Budhrani PH, Farias JR, Shelton MM, Moscoso MS, Park JY, Kip KE (2015) The effects of mindfulnessbased stress reduction on objective and subjective sleep parameters in women with breast cancer: a randomized controlled trial. Psychooncology 24, 424-32. [Medline] [CrossRef] 